mentary action of two or more individuals. The universal experience is that no single isolated compound has ever been found to exhibit unmistakable antineuritic activity. 'To what extent this evidence supports the latter view cannot be stated.

This apparent complex character of all vitamin fractions, so far obtained, accounts for the exceptional difficulty encountered in all efforts to identify the active substance.

\title{
Summary
}

A method is described for the isolation of highly active vitamin fractions from yeast by utilizing the adsorptive power of fuller's earth. The "activated solid" thus obtained is extracted with barium hydroxide solution and the barium eliminated by acidifying with sulfuric acid. The extract, after concentration by vacuum distillation, is precipitated successively with silver nitrate and ammoniacal silver nitrate. Approximately $1 / 3$ of the solids of the extract is precipitated as silver compounds and these contain somewhat more than $1 / 2$ of the antineuritic vitamin. 'The incomplete precipitation of the vitamin base is believed to be due to the considerable solubility of its silver compound.

'The vitamin fractions were found to be quite stable both in solution and in the dried condition. 'They dialyze almost completely through a collodion membrane, and physiological tests showed that all of the vitamin is in the diffusate, thus indicating that the vitamin molecule is of relatively simple constitution. Using nitrogen determinations as a criterion of purity, it was concluded that the highly active fractions contain vitamin and one or more analogous nitrogenous bases, and these cannot be advantageously separated by silver precipitation.

WASHINGTON, D. C.

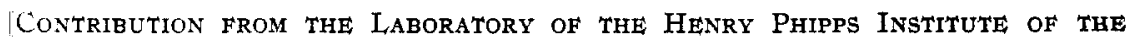
UNIVERSity OF PENNSYlvania]

\section{A SULFONATED NAPHTHYLARSINIC ACID}

\author{
By A. Elizabeth Hill and A. K. Balls
}

Received June 12, 1922

In the course of a certain chemotherapeutic research at this laboratory, it seemed advisable to prepare a compound similar in properties to the $\alpha$-naphthylarsinic acid described by $\mathrm{W}$. Kelbe ${ }^{1}$ but more soluble in water. It was reasonable to suppose that this might be obtained by sulfonating the original $\alpha$-naphthylarsinic acid. A careful review of the literature leads us to believe that only one compound containing both sulfonic and arsenic acid groups on the same nucleus has been prepared. This is the trisulfonic

1 Kelbe, Ber., 11, 1503 (1878). 
acid of triphenyl-arsenic oxide. ${ }^{2}$ Jacobs and Heidelberger ${ }^{3}$ have made other arsenic compounds with both an arsenated and a sulfonated nucleus.

For the purpose of obtaining conveniently a quantity of $\alpha$-naphthylarsinic acid to carry out these experiments, a new method was used which is merely an adaptation of the reaction of $\mathrm{Bart},{ }^{4}$ but which, by giving a product of very good quality and by avoiding the preparation, chlorination, and hydrolysis of mercury dinaphthyl, seemed quite suitable to our needs.

Preparation of $\alpha$-Naphthylarsinic Acid from Napthylamine and Sodium Arsenite. -One mole of $\alpha$-naphthylamine is dissolved in a mixture of $3000 \mathrm{cc}$. of hot water and $100 \mathrm{cc}$. of conc. hydrochloric acid. After cooling to $0^{\circ}, 200 \mathrm{cc}$. of hydrochloric acid (sp. gr. 1.18) is added and the $\alpha$-naphthylamine diazotized in the usual manner with one mole of sodium nitrite. The diazo solution thus obtained is poured into a solution of $600 \mathrm{~g}$. of anhydrous sodium arsenite in $2000 \mathrm{cc}$. of water. ${ }^{.}$

The best yield was obtained by allowing the diazonium compound to decompose slowly at room temperature. The evolution of nitrogen occupies several days, and when it is complete the liquid is filtered from the brown by-product, and then acidified with hydrochloric acid. The precipitate of crude $\alpha$-naphthylarsinic acid is filtered off and purified by dissolving in a cold conc. solution of sodium bicarbonate, the insoluble residue filtered out, and naphthylarsinic acid reprecipitated with hydrochloric acid. By recrystallization from water the substance may be purified still further.

On cooling the aqueous solution, the acid crystallizes in a creamy-white mass of needles, melting at $197^{\circ}$. It is soluble in hot water, ethyl and methyl alcohol, and acetone and is insoluble in mineral acids, benzene, carbon disulfide, carbon tetrachloride, chloroform, toluene and xylene. An analysis of material recrystallized from water gave the following results, also showing the material to be free from water of crystallization.

Analyses: Calc for $\mathrm{C}_{10} \mathrm{HI}_{7} \mathrm{AsO}(\mathrm{OH})_{2}: \mathrm{C}, 47.62 ; \mathrm{H}, 3.60$; As, 29.70 . Found: $\mathrm{C}$, $47.45 ; \mathrm{H}, 4.03 ; \mathrm{As}, 29.69$.

\section{Preparation of Naphthylarsinic Monosulfonic Acid from Naphthylarsinic Acid}

The naphthylarsinic acid prepared as above cannot be sulfonated by employing either diluted or ordinary $98 \%$ acid, even though maintained at high temperatures for a long time. The addition of mercury is likewise without effect.

By using fuming (20\%) sulfuric acid however, the reaction takes place at once and without the use of external heat or catalyst. A considerable evolution of heat occurs during the reaction which is quite rapid, and accompanied by the immediate separation of the monosulfonic acid in solid form.

${ }^{2}$ Michaelis, Ann., 321, 186 (1902).

${ }^{3}$ Jacobs and Heidelberger, This Journal, 41, 1610 (1919).

4 Bart, Ger pat. 250,264, Eng. pat. 568, U. S. pat. 1,061,587.

${ }^{5}$ Using copper powder as a catalyst, the diazo solution is poured into a solution of $192 \mathrm{~g}$. of sodium arsenite in $2000 \mathrm{cc}$. of water, $200 \mathrm{cc}$. of $40 \%$ sodium hydroxide and 150 g. of copper powder, the subsequent procedure being the same in either case. The yield was $30 \mathrm{~g}$. of $\alpha$-naphthylarsinic acid, using copper powder, and $50 \mathrm{~g}$. by the method as described above. We see no advantage in the copper powder procedure. 
One hundred and fifty cc. of fuming sulfuric acid (sp. gr. 1.9) is poured on $50 \mathrm{~g}$. of naphthylarsinic acid and the mixture stirred; the temperature rises to $80-100^{\circ}$. After stirring has been continued for 5 minutes the temperature falls to about $75^{\circ}$ and a gray solid begins to separate. After an hour's time during which the stirring is continued, the mixture (now at room temperature) contains a great amount of solid. It is poured into ice and water (2 liters), also stirred, and after a few minutes the precipitate filtered off. The solid is then best dissolved with sodium hydroxide and, after boiling with charcoal and filtering, the sulfonic acid is precipitated with hydrochloric acid. It can then be recrystallized from boiling water. If the sulfonic acid contains alkaline earths, as is sometimes the case with the use of impure fuming acid, a considerable residue remains insoluble in soda. This getnerally consists of calcium and magnesium salts of the sulfonic acid and can be conveniently decomposed with hydrochloric acid. The free acid, precipitated on cooling the solution, is filtered off and added to the rest. The yield is about $60 \%$ of that theoretically obtainable.

The material has the property, rare among sulfonic acids, of being easily crystallizable, and is capable of being prepared in an almost perfect state of purity. It crystallizes from water in gleaming white plates, is somewhat hygroscopic, and holds its water rather tenaciously. It can be dried in a vacuum or at $100^{\circ}$; no definite amount of water of crystallization was discovered, however. It is easily soluble in hot water, alkalies, acetone, methyl and ethyl alcohol, is difficultly soluble in ether, and is insoluble in mineral acids, benzene, carbon disulfide, carbon tetrachloride, chloroform, toluene, and xylene. It neither melts nor decomposes below $250^{\circ}$.

Calc. for $\mathrm{C}_{10} \mathrm{H}_{7} \mathrm{AsO}(\mathrm{OH})_{2} \mathrm{SO}_{3} \mathrm{H}: \mathrm{C}, 36.13 ; \mathrm{H}, 2.73 ; \mathrm{S}, 9.66 ; \mathrm{As}, 22.57$. Found: C, $35.8 ; \mathrm{H}, 2.54 ; \mathrm{S}, 9.53 ; \mathrm{As}, 22.30$.

Alkaline potassium permanganate easily oxidizes the material. After acidifying and removing of the excess of permanganate, a mixture of substances remains easily soluble in both water and alcohol and, therefore, extracted with alcohol from the evaporated residue. The alcohol extracts a thick yellow hygroscopic liquid which contains both arsenic and sulfur, and gives fluorescence tests for the phthalic acid configuration with resorcin and sulfuric acid, showing its derivation from then aphthalene nuclets. Analysis of this liquid showed water, $4.08 \%$; arsenic, $15.90 \%$; and sulfur, $6.75 \%$.

It will be seen that the arsenic and sulfur are in an equimolecular ratio, which again confirms the observation that the original substance is a monostilfonated naphthylarsinic acid.

This behavior on oxidation would no doubt furnish evidence of value in locating the position of the sulfonic acid group on the naphthalene nucleus. It must be remembered, however, that the material obtained from the oxidation is a viscous liquid without a definite melting point, and with no other definite criterion of a pure compound. It may, therefore, well be a mixture of several substances resulting from the oxidation. On mixing the oily liquid with water, an acid solution results. A few attempts to prepare crystalline salts from the substance were made unsuccessfully, and of course 
until this is done, nothing can be said in this connection concerning the location of the sulfonic group acid.

Three potassium salts have been prepared from the sulfonic acid by adding the calculated quantities of potassium carbonate. The monopotassium salt, the only one recrystallized for analytical purposes, showed arsenic, $20.19 \%$, and potassium, $10.86 \%$, which compare quite closely with values calculated for a monopotassium salt of naphthylarsinic monosulfonic acid, namely, arsenic, $20.25 \%$, and potassium, $10.53 \%$.

Potassium was determined by dissolving the potassium salt in water, precipitating the greater part of the sulfonic acid with strong hydrochloric acid. After filtering this off and washing with dil. hydrochloric acid the potassium was easily determined in the filtrate by the perchlorate method, ${ }^{6}$ finally washing the potassium perchlorate with $98 \%$ alcohol containing a little perchloric acid.

The small amounts of sulfonic acid not precipitated by the hydrochloric acid are oxidized by perchloric acid to alcohol-soluble substances. Control mixtures of 0.4660 and $0.4712 \mathrm{~g}$. of pure potassium carbonate with $0.5 \mathrm{~g}$. free sulfonic acid, respectively, were run to test the applicability of the method; weights of potassium added as carbonate were 0.4660 and 0.4712 g., the weights recovered as perchlorate 0.4652 and $0.4710 \mathrm{~g}$., respectively.

The method is interesting as affording a very easy method for the determination of potassium in a few classes of organic compounds where the oxidation products formed by perchloric acid are soluble in alcohol.

All the potassium salts are white crystalline solids easily soluble in water.

\section{Summary}

1. $\alpha$-Naphthylarsinic acid has been prepared by diazotizing $\alpha$-naphthylamine and then treating with sodium arsenite.

2. Upon treatment of $\alpha$-naphthylarsinic acid with fuming sulfuric acid a monosulfonic acid of $\alpha$-naphthylarsinic acid is produced.

Philadelphia, Pennsyluania

' Morgan, J. Ind. Eng. Chem., 13, 225 (1921). 